\title{
Separation of target structure and medium propagation effects in high-harmonic generation
}

\author{
Cheng Jin ${ }^{1}$, Hans Jakob Wörner ${ }^{2,3}$, V Tosa ${ }^{4}$, Anh-Thu Le ${ }^{1}$, \\ Julien B Bertrand ${ }^{2}$, R R Lucchese ${ }^{5}$, P B Corkum ${ }^{2}$, D M Villeneuve ${ }^{2}$ and \\ C D Lin ${ }^{1}$ \\ ${ }^{1}$ J R Macdonald Laboratory, Physics Department, Kansas State University, Manhattan, KS 66506-2604, \\ USA \\ 2 Joint Laboratory for Attosecond Science, National Research Council of Canada and University of \\ Ottawa, 100 Sussex Drive, Ottawa, Ontario K1A 0R6, Canada \\ ${ }^{3}$ Laboratorium für Physikalische Chemie, ETH Zürich, Wolfgang-Pauli-Strasse 10, 8093 Zürich, \\ Switzerland \\ ${ }^{4}$ National Institute for R\&D of Isotopic and Molecular Technologies, 400293 Cluj-Napoca, Romania \\ 5 Department of Chemistry, Texas A\&M University, College Station, TX 77843-3255, USA \\ E-mail: cjin@phys.ksu.edu
}

Received 16 December 2010, in final form 24 March 2011

Published 20 April 2011

Online at stacks.iop.org/JPhysB/44/095601

\begin{abstract}
We calculate high-harmonic generation (HHG) by intense infrared lasers in atoms and molecules with the inclusion of macroscopic propagation of the harmonics in the gas medium. We show that the observed experimental spectra can be accurately reproduced theoretically despite the sensitivities of the HHG spectra to the experimental conditions. We further demonstrate that the simulated (or experimental) HHG spectra can be factored out as a product of a 'macroscopic wave packet' and the photo-recombination transition dipole moment where the former depends on the laser properties and the experimental conditions, while the latter is the property of the target only. The factorization makes it possible to extract target structure from experimental HHG spectra, and for ultrafast dynamic imaging of transient molecules.
\end{abstract}

(Some figures in this article are in colour only in the electronic version)

High-harmonic generation (HHG) has been employed to probe electronic structure of molecules on an ultrafast time scale in recent years [1-3]. When molecules are placed in an intense laser field, electrons that are removed earlier may be driven back to recollide with the parent ion. HHG occurs when the returning electrons recombine with the parent ion with the emission of high-energy photons as in an inverse photoionization (PI) process. Since PI is a sensitive tool for probing electronic structure of molecules, HHG may serve likewise, but with the advantage of ultrafast temporal resolution as well as covering a coherent broad spectral range from XUV to soft x-rays. Experimentally, however, HHG is generated from all the molecules in the interaction region. The radiations from them co-propagate with the fundamental infrared (IR) beam nonlinearly. To extract structure information of individual molecules, e.g., the amplitude and phase of PI transition dipole from the measured HHG, the propagation effect in the medium should be investigated. For molecular targets, this has not been performed so far. Instead, it was often assumed that HHG was measured under the perfect phase-matching conditions and that the observed harmonics were directly proportional to the harmonics from a single molecule. While such assumptions may be adequate for explaining many experimental observations qualitatively, such as the dependence of HHG on molecular alignment and on symmetry of the molecular orbital, the two-centre interference $[4,5]$, and multiple-orbital contributions to HHG [6], they are inadequate if accurate structure information of individual molecules is to be extracted from the observed HHG spectra. 


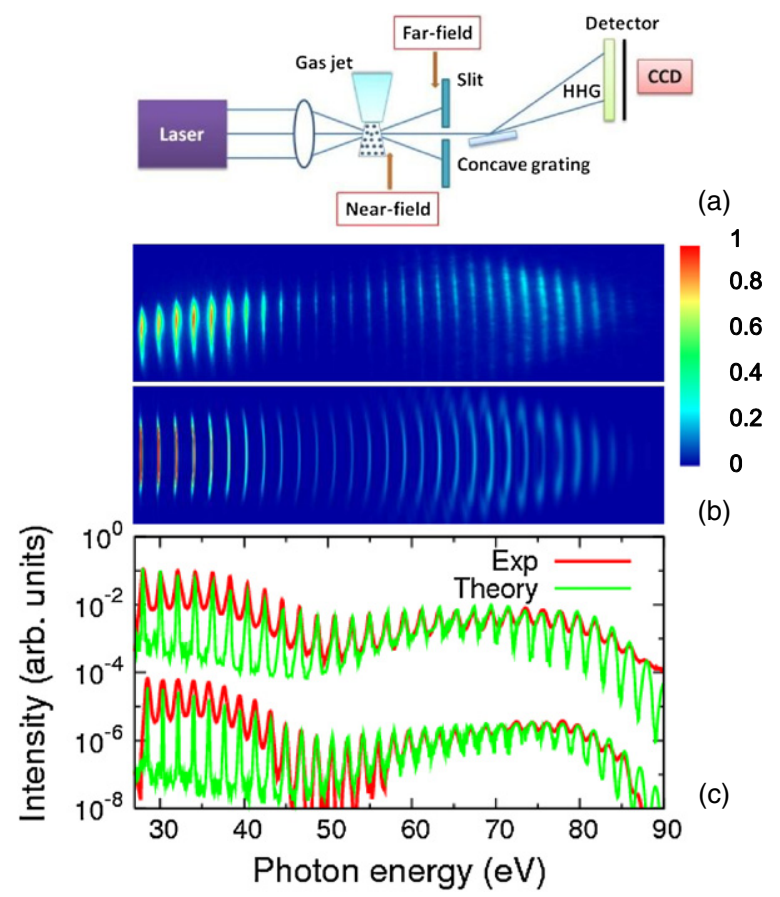

Figure 1. (a) Typical configuration for measuring HHG in the far field. (b) HHG spectra of Ar generated by a $1200 \mathrm{~nm}$ laser. Upper frame: experiment; lower frame: theory. (c) Comparison of theoretical (green curves) and experimental (red curves) HHG yields integrated over the vertical dimension for $1200 \mathrm{~nm}$ (upper curves) and $1360 \mathrm{~nm}$ (lower curves) lasers. Laser parameters are given in the text.

The effect of macroscopic propagation on the observed HHG spectra for atoms has been investigated extensively in the past two decades in connection with the generation of attosecond pulses, see [7, 8]. Maxwell's equations that govern the propagation of the fundamental driving IR field and the generated harmonics are well established. To carry out such propagation calculations, accurately induced atomic dipoles generated by lasers for hundreds of peak intensities should be calculated which serve as the source term of the harmonics. These induced dipoles are often calculated using the strongfield approximation (SFA), or the so-called Lewenstein model [9]. The SFA does not describe the laser-atom interactions accurately; thus, the results from the propagation can only be used to qualitatively interpret experiments. While accurately induced dipoles can be obtained from solving the timedependent Schrödinger equation (TDSE), the calculation is rather time consuming and is rarely attempted except for a very few occasions [10]. Thus after two decades, our understanding of experimental HHG data is still mostly at the qualitative level.

In this paper, we show that such limitations have been removed. We generate HHG spectra theoretically under experimental conditions and the results are compared directly to the observed data. The simulated spectra agree well with the measured one, over a broad photon energy region. The experiments were taken in a geometry as depicted in figure 1(a), using IR laser pulses with wavelengths of $1200 \mathrm{~nm}$ and $1360 \mathrm{~nm}$, respectively, generated in a high-energy optical parametric amplifier (HE-TOPAS). The TOPAS was pumped by the output of a multipass femtosecond amplifier system
$(8 \mathrm{~mJ}, 32 \mathrm{fs}, 50 \mathrm{~Hz})$. High-harmonic radiation generated from the gas jet was allowed to propagate and a slit was placed downstream. After the slit, a concave grating dispersed the harmonics which were then detected with a CCD detector. The gas jet was formed from a supersonic expansion of $\mathrm{Ar}$ or $\mathrm{N}_{2}$ at a stagnation pressure of 3 bars. Experiments were performed on $\mathrm{Ar}$ and on either isotropic or aligned $\mathrm{N}_{2}$ molecules. The experimental spectra were corrected for the response of the grating and detector.

Figure 1(b) shows the HHG spectra of Ar generated by a $1200 \mathrm{~nm}$ laser. The horizontal axis is the photon energy and the vertical axis is the transverse spatial dimension. The upper frame is from the measurement, while the bottom frame is from the simulation. The two spectra are normalized to each other at harmonic 75 , or at photon energy of $77 \mathrm{eV}$. There is a general agreement between the two spectra. The 'up-down' asymmetry in the experimental HHG spectra is due to asymmetry in the laser beam profile. The faint features near $50 \mathrm{eV}$ are the 'famous' Cooper minimum in Ar [11], observed in PI, as well as in earlier HHG spectra [12-14]. The harmonic yields integrated over the vertical dimension are compared in the upper half of figure 1(c). The lower half shows the HHG spectra taken with the $1360 \mathrm{~nm}$ laser. In both cases, we can see very good agreement between theory and experiment over the $30-90 \mathrm{eV}$ region covered. Experimentally, the gas jet is $0.5 \mathrm{~mm}$ long and placed $3 \mathrm{~mm}$ after the focus. A vertical slit with a diameter of $100 \mu \mathrm{m}$ is placed $24 \mathrm{~cm}$ after the gas jet. For the $1200(1360) \mathrm{nm}$ the beam waist at the laser focus is $47.5(52.5) \mu \mathrm{m}$, and the pulse duration is $\sim 40(\sim 50)$ fs. To achieve the best overall agreement, in the simulation the peak intensity and gas pressure for each wavelength are adjusted until best overall fit of the data is achieved. Thus for the $1200 \mathrm{~nm}$ laser, the peak intensity for experiment (theory) is $1.6(1.5) \times 10^{14} \mathrm{~W} \mathrm{~cm}^{-2}$, and the gas pressure is 28 (84) Torr. For the $1360 \mathrm{~nm}$ laser, the corresponding parameters are $1.25(1.15) \times 10^{14} \mathrm{~W} \mathrm{~cm}^{-2}$, and 28 (56) Torr, respectively. By using a higher pressure in the simulation, we find that the higher harmonics become sharper, as in experiments. The (normalized) envelope of the harmonic spectra, however, does not depend much on the gas pressure, see figure 3(b).

In the theoretical simulation, we first obtain singleatom-induced dipole using the quantitative rescattering (QRS) theory [15-17]. The Ar is treated in the single-active electron approximation using the model potential proposed by Muller [18]. The resulting induced dipoles for different peak intensities are then fed into Maxwell's equations. The propagation equations for the fundamental field and the harmonics are the standard ones [19-21]. For Ar target, we include dispersion, absorption, Kerr and plasma effects on the fundamental field in the medium. For the harmonics, only the dispersion and absorption are included. The harmonic yields emitted at the exit face of the gas jet (near field) are propagated to the far field where the harmonics are measured. They are obtained from the near-field harmonics through a Hankel transform [22, 23]. We assume the laser beam in the entrance of gas jet has the Gaussian shape.

A careful examination of figures 1(b) and (c) reveals that there are still small discrepancies between the experimental 


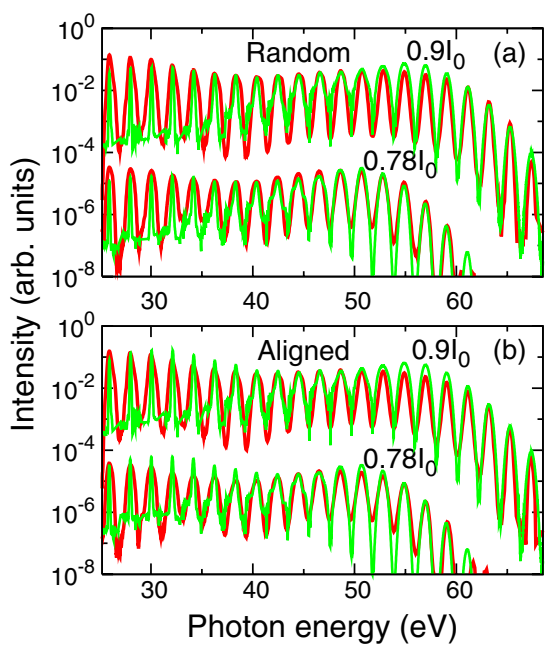

Figure 2. Comparison of HHG spectra from theory (green curves) and experimental results (red curves) of [27], (a) for randomly distributed $\mathrm{N}_{2}$ and (b) for $\mathrm{N}_{2}$ aligned along the laser polarization direction. The laser intensities are indicated where $I_{0}=10^{14} \mathrm{~W}$ $\mathrm{cm}^{-2}$. See the text for additional laser parameters.

data and the simulation. The harmonic width (or harmonic chirp) is narrower from the theory than from the experiment. Harmonic chirp is a direct consequence of temporal variation of laser intensity. The harmonic width is mainly influenced by the pulse duration, pressure and laser intensity [24-26]. The width decreases with increasing pulse duration, and with decreasing gas pressure. Other experimental factors like use of the slit and location of the detector also can affect the HHG spectra.

High-order harmonics from molecules by $1200 \mathrm{~nm}$ lasers have been reported for aligned and randomly distributed $\mathrm{N}_{2}$ and $\mathrm{CO}_{2}$ recently [27]. Here, we report our simulated results for $\mathrm{N}_{2}$, at the two peak laser intensities, 0.9 and $1.1 \times 10^{14} \mathrm{~W} \mathrm{~cm} \mathrm{~cm}^{-2}$, reported in [27]. To achieve good agreement in the cutoff positions, the two intensities used in the theory are 0.78 and $0.9 \times 10^{14} \mathrm{~W} \mathrm{~cm}^{-2}$ instead, respectively. Since the experiment was carried out at low laser intensity and low gas pressure, the harmonics are propagated without absorption and dispersion effects from the medium, and the fundamental laser field is not modified through the medium [28]. In the theoretical simulation, we first obtain induced dipoles of fixed-in-space molecules using QRS theory [16, 17] for different laser peak intensities. The induced dipoles are averaged coherently according to the alignment distribution and then fed into Maxwell's equations. Figure 2 shows the good overall agreement between the measured and the simulated spectra, for both randomly distributed and aligned $\mathrm{N}_{2}$. By examining the experimental HHG spectra more carefully, they reveal a shallow minimum at $38 \pm 2 \mathrm{eV}$ (low intensity) and at $41 \pm 2 \mathrm{eV}$ (high intensity) for both aligned and unaligned molecules. The theory also predicts a minimum: for unaligned molecules, the minimum is at $\sim 39 \mathrm{eV}$ for low intensity and $\sim 40 \mathrm{eV}$ for high intensity. For aligned molecules, the minimum is at $\sim 42 \mathrm{eV}$ for low intensity and $\sim 44 \mathrm{eV}$ for high intensity. In the experiment, the degree of alignment was estimated to be $\left\langle\cos ^{2} \theta\right\rangle=0.6-0.65$. In the simulation, an alignment distribution of $\cos ^{4} \theta$ is used. Note that only HOMO is included in the calculation. We believe that this is the first time that HHG spectra from molecules have been calculated including the propagation effect in the medium and the simulated results have been compared directly to the measured spectra. In the future, HHG spectra taken at different alignment angles should be compared together. Such comparison would help to identify factors that contribute to the remaining discrepancies between experiment and simulation.

The macroscopic HHG spectra can be expressed as [28]

$$
S_{h}(\omega) \propto \omega^{4}|W(\omega)|^{2}|d(\omega)|^{2}
$$

where $W(\omega)$ (the complex amplitude) is called 'macroscopic wave packet' (MWP), and $d(\omega)$ is the PI transition dipole moment for the atom. Note that the tunnelling ionization rate of the target enters into equation (1) as a proportional constant. For the molecule $d(\omega)$ is taken to be the coherently averaged PI transition dipole moment $d^{\text {avg }}(\omega)=$ $\int_{0}^{\pi} N(\theta)^{\frac{1}{2}} \rho(\theta) d(\theta, \omega) \sin \theta \mathrm{d} \theta$, where $N(\theta)$ is the alignmentdependent ionization probability, $\rho(\theta)$ is the alignment distribution and $d(\theta, \omega)$ is the parallel component of the alignment-dependent transition dipole moment [29, 30]. The polarization of the pump laser is assumed parallel to the probe laser. For unaligned molecules, $\rho(\theta)$ is a constant. Actually, MWP has the clear physical meaning. It can be considered as the collective effect of microscopic wave packets for the returning electrons $[16,17]$, which is governed by Maxwell's equations. In other words, the laser and macroscopic medium effects are all combined into MWP.

The validity of equation (1) has been checked in Jin et al [28] when both the laser intensity and the gas pressure are low. The correctness of this relation has been assumed in Itatani et al [1] by comparing Ar with $\mathrm{N}_{2}$, and in Levesque et al [31] for rare gas atoms. However, this relation has not been carefully checked for different focusing conditions and laser parameters. Theoretically, we have checked the validity of equation (1) carefully. We carried out macroscopic propagation calculation of HHG using single-atom (single-molecule)-induced dipole obtained by QRS and SFA. We have been able to show that the MWP obtained from the two calculations agrees rather well, irrespective of laser parameters or the focusing conditions. In other words, the medium propagation only affects HHG through its modifications on the MWP. In this way, to study propagation effect on HHG, we can just study how the MWP (only the amplitude is considered below) depends on the lasers and the experimental conditions.

In figure 3(a) we show the dependence of MWP on the position of the Ar gas jet with respect to the laser focus. The laser intensity is $1.6 \times 10^{14} \mathrm{~W} \mathrm{~cm}^{-2}$, and the gas pressure is 56 Torr. For easy visualization we show the smooth envelope of $|W(\omega)|$. The three curves are for the gas jet at $z=$ $-3 \mathrm{~mm}$ (gas jet before laser focus), $z=0$ (at), +3 (after). It is generally known that HHG achieves best phase matching if the gas jet is placed behind the laser focus where the dipole phase from the harmonic can be partially cancelled by the Gouy phase. Thus among the three curves, the 'after' curve is 

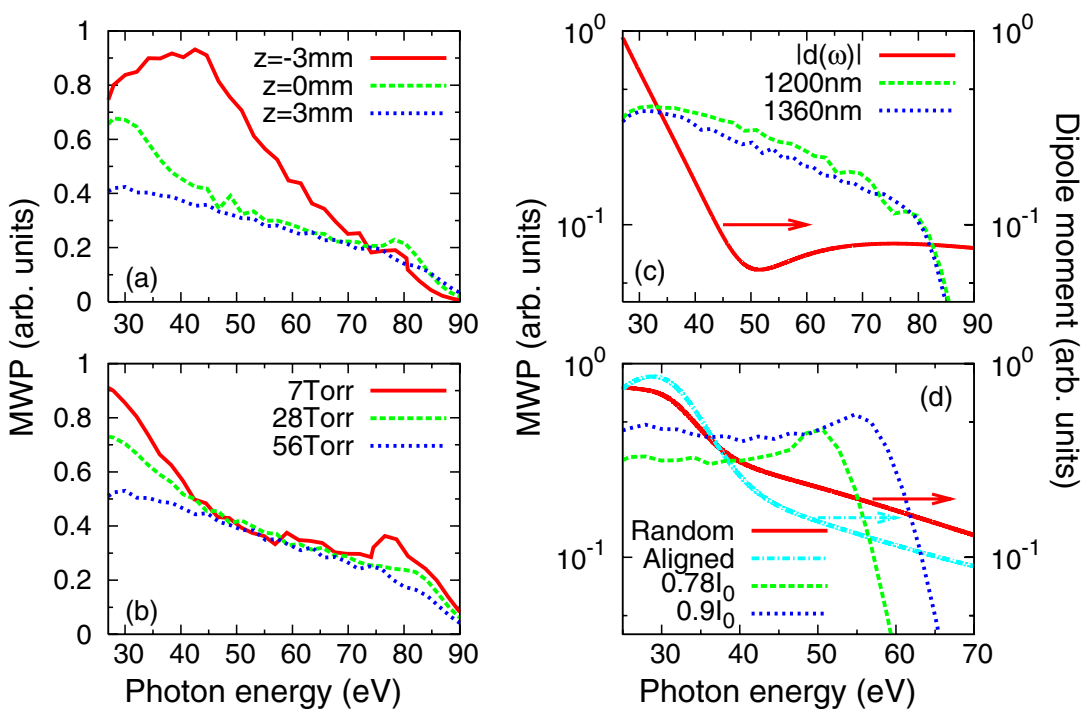

Figure 3. (a) Dependence of macroscopic wave packet $|W(\omega)|$ (MWP) on the position of the Ar gas jet with respect to laser focus; (b) Effect of gas pressure on MWP. The curves are renormalized such that they should fall on the same curve if the perfect phase-matching condition is fulfilled. (c) The MWP for the two lasers, and the magnitude of the PI transition dipole moment of Ar. (d) Same as in (c) but for $\mathrm{N}_{2}$. The MWP is for two different laser intensities, and the averaged PI transition dipoles are for isotropic and aligned molecules. The laser intensities are indicated where $I_{0}=10^{14} \mathrm{~W} \mathrm{~cm}^{-2}$. See the text.

the flattest one. For the 'before' focus, the MWP varies most as the photon energy is changed, and the phase (not shown) varies widely from order to order-reflecting poor phase matching for this geometry.

In figure $3(\mathrm{~b})$ we compare the MWP derived from changing the Ar gas pressure for the 'after' focusing condition. The MWP has been normalized by the ratio of the pressure. Under a perfect phase-matching condition, the MWP $|W(\omega)|$ (the amplitude) should be proportional to the pressure [32, 33]. The three curves are on top of each other from 45 to $75 \mathrm{eV}$, indicating good phase matching in this energy region, but differ somewhat at lower and higher energies, indicating that a good phase-matching condition is not fulfilled. This demonstrates that the phase-matching condition cannot be achieved for all the harmonics in a given experiment.

According to equation (1), the minimum in the HHG spectra can occur for different reasons. In figure 3(c), the MWPs derived from the Ar target using $1200 \mathrm{~nm}$ and $1360 \mathrm{~nm}$ lasers are shown. The two MWPs are quite similar but near $50 \mathrm{eV}$, they have slight different slopes. On the other hand, the PI transition dipole reveals a clear but broad Cooper minimum near $50 \mathrm{eV}$. Thus, the broad minimum in the HHG spectra shown in figure 1(c) is due to the minimum in the PI transition dipole. To pin down the position of the 'real' minimum, on the other hand, is not as easy since the minimum position can be modified somewhat by the MWP.

A similar analysis can be carried out on the HHG spectra of $\mathrm{N}_{2}$ shown in figure 2. The averaged PI transition dipole indeed shows a rapid drop near $40 \mathrm{eV}$, which is due to the presence of a shape resonance [34] of $\mathrm{N}_{2}$ in the lower energy. The rapid drop is more pronounced for aligned molecules than for random ones, see figure 3(d). For the MWP, under the same laser intensity, we have checked that they are the same for randomly distributed and aligned molecules. Thus, it explains why the HHG from single-molecule response can be used to interpret how the intensity of each harmonic changes with pump-probe time delay in Le et al [35]. However, the MWP changes more rapidly with laser intensity, especially for the longer wavelength laser used here. We note that the two MWPs in figure 3(d) have somewhat different slopes near $40 \mathrm{eV}$. The multiplication of the MWP and the PI transition dipole results in a weak minimum in the observed HHG spectra. The minimum would be more clearly seen if the molecules were better aligned. From Le et al [16], the minimum in PI transition dipole changes rapidly with the alignment angle and the effect is severely averaged out when molecules are not well aligned. We further mention that the MWPs in figures 3(c) and (d) are rather different. They are due to the large difference in the laser peak intensities used [28]. In the future, it is desirable that predictions such as those in figure 3 be checked experimentally.

In summary, we have demonstrated that experimental HHG spectra can now be accurately reproduced theoretically. The theory starts with the calculation of laser-induced dipole from single atom or molecule using the recently developed QRS theory [16, 17]. The propagation effect of the fundamental field and the harmonics in the medium is incorporated by solving Maxwell's equations. We have further shown that the simulated (and experimental) HHG spectra can be expressed as the product of a MWP and the photorecombination transition dipole moment. The latter is a property of the target, and is independent of the lasers, not of the propagation effect. This factorization makes it possible to extract target structure information from the experimental HHG spectra. It provides the needed theoretical basis for using HHG as ultrafast probes of excited molecules, such as those demonstrated recently [3]. Clearly, this work also opens up opportunities for the quantitative studies of the phases of HHG which are fundamental to the generation of attosecond pulses. 


\section{Acknowledgments}

This work was supported in part by Chemical Sciences, Geosciences and Biosciences Division, Office of Basic Energy Sciences, Office of Science, US Department of Energy.

Note added. During the submission of this paper, our detailed theory paper on this subject has appeared, see [36]. Furthermore, a similar study on the HHG spectra of Ar has appeared, see [37].

\section{References}

[1] Itatani J, Levesque J, Zeidler D, Niikura H, Pépin H, Kieffer J C, Corkum P B and Villeneuve D M 2004 Nature $\mathbf{4 3 2} 867$

[2] Smirnova O, Mairesse Y, Patchkovskii S, Dudovich N Villeneuve D, Corkum P and Ivanov M Yu 2009 Nature 460972

[3] Wörner H J, Bertrand J B, Kartashov D V, Corkum P B and Villeneuve D M 2010 Nature 466604

[4] Kanai T, Minemoto S and Sakai H 2005 Nature 435470

[5] Lein M, Hay N, Velotta R, Marangos J P and Knight P L 2002 Phys. Rev. Lett. 88183903

[6] McFarland B K, Farrell J P, Bucksbaum P H and Gühr M 2008 Science 3221232

[7] Brabec T and Krausz F 2000 Rev. Mod. Phys. 72545

[8] Krausz F and Ivanov M 2009 Rev. Mod. Phys. 81163

[9] Lewenstein M, Balcou Ph, Ivanov M Yu, L'Huillier A and Corkum P B 1994 Phys. Rev. A 492117

[10] Gaarde M B, Murakami M and Kienberger R 2006 Phys. Rev. A 74053401

[11] Cooper J W 1962 Phys. Rev. 128681

[12] Wörner H J, Niikura H, Bertrand J B, Corkum P B and Villeneuve D M 2009 Phys. Rev. Lett. 102103901

[13] Minemoto S, Umegaki T, Oguchi Y, Morishita T, Le A T, Watanabe S and Sakai H 2008 Phys. Rev. A 78061402

[14] Colosimo P et al 2008 Nat. Phys. 4386
[15] Morishita T, Le A T, Chen Z and Lin C D 2008 Phys. Rev. Lett. 100013903

[16] Le A T, Lucchese R R, Tonzani S, Morishita T and Lin C D 2009 Phys. Rev. A 80013401

[17] Lin C D, Le A T, Chen Z, Morishita T and Lucchese R R 2010 J. Phys. B: At. Mol. Opt. Phys. 43122001

[18] Muller H G 1999 Phys. Rev. A 601341

[19] Priori E et al 2000 Phys. Rev. A 61063801

[20] Gaarde M B, Tate J L and Schafer K J 2008 J. Phys. B: At. Mol. Opt. Phys. 41132001

[21] Tosa V, Kim H T, Kim I J and Nam C H 2005 Phys. Rev. A 71063807

[22] L'Huillier A, Balcou Ph, Candel S, Schafer K J and Kulander K C 1992 Phys. Rev. A 462778

[23] Tosa V, Kim K T and Nam C H 2009 Phys. Rev. A 79043828

[24] Gaarde M B, Salin F, Constant E, Balcou Ph, Schafer K J, Kulander K C and L'Huillier A 1999 Phys. Rev. A 591367

[25] Zaï A et al 2008 Phys. Rev. Lett. 100143902

[26] He X et al 2009 Phys. Rev. A 79063829

[27] Wörner H J, Bertrand J B, Hockett P, Corkum P B and Villeneuve D M 2010 Phys. Rev. Lett. 104233904

[28] Jin C, Le A T and Lin C D 2009 Phys. Rev. A 79053413

[29] Le A T, Lucchese R R and Lin C D 2010 Phys. Rev. A 82023814

[30] Jin C, Le A T, Zhao S F, Lucchese R R and Lin C D 2010 Phys. Rev. A 81033421

[31] Levesque J, Zeidler D, Marangos J P, Corkum P B and Villeneuve D M 2007 Phys. Rev. Lett. 98183903

[32] Yakovlev V S, Ivanov M and Krausz F 2007 Opt. Express 1515351

[33] Shiner A D, Trallero-Herrero C, Kajumba N, Bandulet H-C, Comtois D, Légaré F, Giguère M, Kieffer J-C, Corkum P B and Villeneuve D M 2009 Phys. Rev. Lett. 103073902

[34] Lucchese R R, Raseev G and McKoy V 1982 Phys. Rev. A 252572

[35] Le A T, Lucchese R R, Lee M T and Lin C D 2009 Phys. Rev. Lett. 102203001

[36] Jin C, Le A T and Lin C D 2011 Phys. Rev. A 83023411

[37] Farrell J P, Spector L S, McFarland B K, Bucksbaum P H, Gühr M, Gaarde M B and Schafer K J 2011 Phys. Rev. A 83023420 